\title{
What's for dinner?: Undescribed species of porcini in a commercial packet
}

Accurate diagnosis of the components of our food and a standard lexicon for clear communication is essential for regulating global food trade and identifying food frauds.

Reliable identification of wild collected foods can be particularly difficult, especially when they originate in under-documented regions or belong to poorly known groups such as Fungi. Porcini, one of the most widely traded wild edible mushrooms in the world, are large and conspicuous and they are used as a food both on their own and in processed food products. China is a major exporter of porcini, most of it ending up in Europe. We used DNAsequencing to identify three species of mushroom contained within a commercial packet of dried Chinese porcini purchased in London. Surprisingly, all three have never been formally described by science and required new scientific names. This demonstrates the ubiquity of unknown fungal diversity even in widely traded commercial food products from one of the most charismatic and least overlooked groups of mushrooms. Our rapid analysis and description makes it possible to reliably identify these species, allowing their harvest to be monitored and their presence tracked in the food chain. 
1 Bryn T.M. Dentinger

2 Mycology Section, Jodrell Laboratory, Royal Botanic Gardens, Kew, Richmond, Surrey, TW9

3 3DS, UK

4 Laura M. Suz

5 Mycology Section, Jodrell Laboratory, Royal Botanic Gardens, Kew, Richmond, Surrey, TW9

$63 \mathrm{DS}, \mathrm{UK}$

7 Corresponding author:

8 Bryn T M Dentinger

9 Jodrell Laboratory, Royal Botanic Gardens, Kew, Richmond, Surrey, TW9 3DS, UK

$10 \quad(020) 83325106$

11 b.dentinger@,kew.org 


\section{Introduction}

13 Kingdom Fungi is one of the most diverse groups of eukaryotes with estimates ranging

14 from 500,000 to nearly 10 million species, yet they remain vastly underdocumented (Bass \&

15 Richards, 2011). Present rates of description, which add on average about 1200 new species

16 annually (Hibbett et al., 2011), are grossly inadequate for the task. Given that human society has

17 derived tremendous benefit from the foods, medicines, and ecological services provided by as

18 little as $1 \%$ of the fungi we know of, the impact of this missing diversity on human livelihoods is

19 potentially profound. Importantly, this missing diversity is not just restricted to remote,

20 underexplored regions of the world, but is a pervasive phenomenon where even our foods can

21 harbor unknown species.

22 Although taxonomists regard new fungal taxa as commonplace, they are often of little

23 apparent consequence to human society and largely go unnoticed by the public. Like all groups of

24 organisms, our knowledge of fungal diversity is biased towards taxa of greatest concern to

25 ourselves, such as edible fungi. For example, wild mushrooms collected and sold as food around

26 the world generally belong to a handful of well-known taxa (e.g. truffles and chanterelles), most

27 of which have long histories of use in European cuisine. However, even some of these well-

28 known groups have been shown to contain underappreciated levels of diversity. One of these,

29 porcini, has recently been shown to be far more diverse than previously thought (Dentinger et al.,

30 2010; Feng et al., 2012), suggesting the potential for unknown species to end up in the

31 international food supply chain. Although no porcini are known to be poisonous, food allergens

32 have been reported from them (Torricelli et al., 1997; Helbling et al., 2002; Castillo et al., 2013).

33 Therefore, insufficient knowledge of the porcini species contained in food products could pose a

34 health concern.

35 Porcini are estimated to have an annual worldwide consumption up to 100,000 metric tons

36 (Hall et al., 1998). However, their harvest is restricted to wild foraging since, to date, their 
37 cultivation has failed. The high prices for this wild food foraged locally in Europe and North

38 America has driven the market towards less costly sources, such as China (Sitta \& Floriani,

39 2008). According to the official website of Yunnan Province (www.yunnan.cn), the major

40 exporter of wild mushrooms in China, locally-sourced porcini have been exported to Europe

41 since 1973, and mushrooms of Chinese origin now account for approximately half of all dried

42 porcini in Italy (Sitta \& Floriani, 2008). The Chinese species of porcini have been shown

43 previously to be more closely related to European Boletus aereus than they are to the core

44 commercial species, $B$. edulis, with which they last shared a common ancestor up to $\sim 56$ million

45 years ago (Dentinger et al., 2010; Feng et al., 2012).

46 Given what was previously known about the commercial porcini originating in China, we

47 hypothesized that the contents of a commercially available packet of porcini in the UK would

48 contain multiple species. We set out to rapidly diagnose these species using molecular-based

49 'turbo-taxonomy' (Butcher et al. 2012) that employs a combination of modern tools and

50 approaches. Our results show that, with a combination of phylogenetic taxonomy and e-published

51 nomenclature, three previously unnamed species of porcini could be quickly recognized and

52 formally named from a single packet sold in a London grocer.

53 Material and Methods

54 A packet of dried porcini was purchased from a grocer in southwest greater London in

55 October 2013. Fifteen pieces of mushroom were removed arbitrarily from the packet and DNA

56 was extracted using the Sigma Extract-N-Amp kit. The full ITS region of the nrDNA was PCR-

57 amplified using primers ITS1F and ITS4 (White et al., 1990; Gardes \& Bruns, 1993). Successful

58 amplicons were purified using ExoSAP-IT (USB, Cleveland, OH) and sequenced bidirectionally

59 using BigDye3.1 with an ABI 3730 (Applied Biosystems, Foster City, CA). Complementary

60 unidirectional reads were aligned and edited using Sequencher 4.2 (GeneCodes, Ann Arbor, MI). 
61 New sequences were combined with 22 related sequences identified using a combination

62 of BLAST searches and the corresponding top hits' putative species clades reported by Dentinger

63 et al. (2010) and Feng et al. (2012). These related sequences were downloaded from GenBank

64 and correspond to “Boletus sp. nov. 2”(EU231965-66; Dentinger et al., 2010)/’Boletus sp. nov.

65 6”(JN563907-08, -09, -11-13, -17; Feng et al., 2012), “Boletus sp. nov. 3”(EU231964; Dentinger

66 et al., 2010)/'Boletus sp. nov. 7’(JQ172782-83, JN563901-06; Feng et al., 2012), and “Boletus

67 sp. nov. 5"(JQ563914-16, -18-19; Feng et al., 2012). A total of 38 ingroup sequences and one

68 outgroup sequence (Boletus aereus, UDB000940) were aligned using MUSCLE (Edgar, 2004) in

69 SeaView v4.4.0 (Galtier, Gouy \& Gautier, 1996) and the terminal gaps converted to missing data.

70 The final matrix consisted of 802 aligned positions, of which 742 were constant and 26 were

71 parsimony uninformative (34 autapomorphic). Minimum and maximum intra- and inter-specific

72 uncorrected "p" distances were calculated using PAUP*v4.0 (Swofford, 2002). A maximum

73 likelihood tree was generated under a GTR + G substitution model using the Pthreads parallelized

74 version of RAxML v7.0.3 (Stamatakis, 2006; Ott et al., 2007) with nonparametric rapid

75 bootstrapping set to automatically terminate with the 'autoMRE' function. A GMYC analysis

76 using the single method (Pons et al., 2006; Fujisawa \& Barraclough, 2013) was conducted with

77 the 'splits' package (v1.0-18) in R version 2.15.0 (R Development Core Team 2009) on an

78 ultrametric tree generated using BEAST v1.8.0 (Drummond et al., 2012). The BEAST analysis

79 applied a rate-smoothing algorithm using an uncorrelated lognormal relaxed clock model

80 (Drummond et al., 2006), the GTR + G substitution model, speciation under a Yule process, the

81 'ucld.mean' prior set to a gamma distribution with a shape of .001 and a scale of 1000 with all

82 other priors set to default values, and 10 million generations sampling every 1000 generations.

83 An ultrametric starting tree was provided using the best ML tree from RAxML with branches

84 transformed using non-parametric rate smoothing in TreeEdit v1.0a10. The perl script Burntrees

85 [Nylander J.A.A., http://www.abc.se/ nylander/burntrees/burntrees.html] was used to sample 
86 every 98 trees from the stationary posterior distribution in the BEAST analysis after the first 250

87 were discarded as the burn-in. These 100 trees were imported for Bayesian GMYC (bGMYC)

88 analysis in R (Reid \& Carstens, 2012). Twenty-six GMYC models were evaluated within the

$8995 \%$ confidence and significant clusters were described as new taxa using the 'turbo-taxonomy'

90 approach (Butcher et al. 2012), facilitated by the rapid e-publishing tool available through Index

91 Fungorum (www.indexfungorum.org). Voucher material was deposited in the fungarium at the

92 Royal Botanic Gardens, Kew (K) and all sequences were submitted to GenBank (KF815926-937, 93 KF854281-283).

94 Results and Discussion

95 The GMYC model with the greatest significant ML score included three ML clusters (1-

9610 clusters with $95 \%$ confidence) plus the root (4 ML entities; $2-23$ with $95 \%$ confidence).

97 GMYC supports for the three ML clusters were weak, low bGMYC posterior probabilities

98 indicated a substantial level of phylogenetic uncertainty, while the maximum likelihood

99 bootstraps supported reciprocal monophyly (79\%, 76\% and 100\% for each cluster respectively;

100 Figure 1). Percent sequence similarity did not support distinction between any of the three species

101 detected by GMYC and bootstrapping, where the minimum uncorrected pairwise distances

102 between clades was greater than the maximum uncorrected pairwise distances within clades

103 (Table 1). This result suggests that, while GMYC may be particularly sensitive to phylogenetic

104 uncertainty as revealed by the low support values, for this dataset it performs better at diagnosing

105 phylogenetic units than the commonly used percent similarity threshold of 97\% (e.g., O'Brien et

106 al. 2005). The phylogenetic uncertainty observed is almost certainly caused by a high ratio of

107 parsimony uninformative variable sites (60 variable positions, 34 parsimony uninformative) to

108 phylogenetically informative changes (26 positions). Of the informative characters, only 11 of

109 them correspond to variable positions between the two closest taxa, B. bainiugan and $B$. 
110 meiweiniuganjun, with five sequences showing heterozygous bases at 6 positions (possibly due to

111 incomplete lineage sorting) and only three of these corresponding to synapomorphic substitutions

112 (Figure 1). Five sequences contained autapomorphic substitutions in 18 positions, representing

113 more than half of all parsimony uninformative characters, with up to 9 autapomorphies occurring

114 in a single sequence (JN563917). These autapomorphies translate into longer terminal branch

115 lengths relative to internal nodes, which reduces the distinction of within and between cluster

116 branching patterns, a phenomenon that is known to affect GMYC supports (Fujisawa \&

117 Barraclough, 2013). These substitutions may indicate true variation in the ITS region, yet $94 \%$

118 comes? from sequences downloaded from GenBank, with only two sequences (JN563906,

119 JN563917) contributing $83 \%$ of the autapomorphies. We suspect that, rather than true variation,

120 these substitutions may instead be the result of sequencing and editing errors. Such errors can

121 have large impacts on phylogenetic inference when the number of phylogenetically informative

122 sites is small, such as in ITS sequences of recently diverged fungi, underscoring the importance

123 of careful scrutiny during sequence preparation.

124 Three species could be identified based on corroboration of ML-supported reciprocal

125 monophyly and GMYC clustering, and these corresponded to lineages previously reported in

126 phylogenetic analyses (Dentinger et al., 2010; Feng et al., 2012, Sitta \& Floriani, 2008), but none

127 of which were formally named or described. Review of recent treatments of Chinese boletes also

128 did not provide names for these taxa, which have been treated as a handful of species that occur

129 in Europe and North America (Zang, 2006). New names were formally published on 12 October

1302013 (see http://www.indexfungorum.org/Publications/Index\%20Fungorum\%20no.29.pdf for

131 terse descriptions ${ }^{1}$, voucher information, and GenBank accessions corresponding to these taxa).

132 We hope that by naming these taxa and providing reference sequences for comparison, we will

11 The numbers reported in the original descriptions should be multiplied by 2.43 to achieve

2 correct measurements of cells and spores. 
133 encourage mycologists with ready access to fresh collections of these species to record and

134 document their characteristics and discover new features that may help to distinguish them.

135 Together with improvements in single-locus diagnosis leading to more robust inferences

136 of evolutionary significant units (Butcher et al., 2012), rapid survey and diagnosis of vast

137 communities of undescribed diversity is initiating a revolution in taxonomy (Riedel et al., 2013).

138 This is particularly true for Fungi, which are hyperdiverse and largely cryptic, requiring indirect

139 detection with environmental sequencing for documenting their true diversity (Taylor et al. 2014,

140 Lücking et al. 2014). As a consequence, a vast quantity of fungal diversity is only known from

141 DNA sequences, and these are accumulating in public databases at incredibly rapid rates (Hibbett

142 et al., 2011). Although recent attempts to accelerate species description using short, unique DNA

143 sequences 'DNA barcoding' (Hebert et al., 2003) and rapid, short description 'turbo-taxonomy'

144 (Butcher et al., 2012) hold promise for meeting the enormous challenge of documenting

145 hyperdiverse and largely unknown groups of organisms (Riedel et al. 2013), they still remain

146 marginal to traditional methods for formal diagnosis of fungal diversity.

147 Turbo-taxonomy is an important improvement to efficiency in reconciling molecular

148 diagnosis with a standard application of names that enable universal communication about

149 biodiversity. Together, DNA sequence-based diagnosis and turbo-taxonomy catalyze description

150 of new species, thereby greatly accelerating the rate at which diversity can be documented and

151 recognized. Although descriptions based on features of organisms that are readily observed

152 without specialized techniques are ideal, this is not always possible and descriptions based on

153 features of DNA sequences could be automated to satisfy rules on naming. Automated pipelines

154 that integrate analysis, taxonomy, and nomenclature will soon accelerate this revolution, enabling

155 us to capture the most comprehensive baseline information on global organismal diversity

156 possible. Given estimated rates of species extinction from $0.1-5 \%$ per year (Costello et al., 2013),

157 and using recent estimates of global fungal diversity of $\sim 6$ million species (Taylor et al., 2014), 
158 extinction rates may exceed description rates in Fungi by up to 5 times. An 'integrative fast track'

159 approach (Riedel et al. 2013) offers the only tractable solution presently available to filling this

160 knowledge gap. And as has been shown here with the three new species of porcini in a widely

161 available commercial product, this knowledge gap can and does have direct impacts on our lives.

\section{Conclusions}

163 Our analysis of 15 pieces of dried porcini mushrooms from a single commercial packet

164 showed three species corresponding to lineages that although previously reported in phylogenetic

165 analyses have never been formally named or described until now. The recognition of these

166 species enables them to be monitored in foods and facilitates countries' adherence to international 167 agreements on exploitation of wildlife, i.e. the Convention on Biological Diversity.

\section{Acknowledgements}

169 We are grateful to Rachel Mason Dentinger, who serendipitously supported this research through

170 a spontaneous purchase of dried porcini for our dinner, and to Paul Kirk for nomenclatural advice

171 and for facilitating the e-publication of the taxonomic treatments cited in this study. Meredith

172 Oyen helped locate and translate the Chinese website. We are also grateful for the comments

173 from the editor and four reviewers that improved this report.

\section{References}

175 Bass D, Richards TA. 2011. Three reasons to re-evaluate fungal diversity 'on Earth and in the 176 ocean'. Fungal Biology Reviews 25:159-164. 
177 Butcher BA, Smith MA, Sharkey MJ, Quicke DLJ. 2012. A turbo-taxonomic study of Thai

178 Aleiodes (Aleiodes) and Aleiodes (Arcaleiodes) (hymenoptera: braconidae: rogadinae) based

179 largely on COI barcoded specimens, with rapid descriptions of 179 new species. Zootaxa 3457:1-

$180 \quad 232$.

181 Castillo C, Lara B, Cruz M-J, Muñoz X. 2013. Protein identification of two allergens of Boletus

182 edulis causing occupational asthma. American Journal of Respiratory and Critical Care

183 Medicine 187:1146-1148.

184 Costello MJ, May RM, Stork NE. 2013. Can we name Earth's species before they go extinct?

185 Science 339:413-416.

186 Dentinger BTM, Ammirati JF, Both EE, Desjardin DE, Halling RE, Henkel TW, Moreau P-A,

187 Nagasawa E, Soytong K, Taylor AF, Watling R, Moncalvo J-M, McLaughlin DJ. 2010. Molecular

188 phylogenetics of porcini mushrooms (Boletus section Boletus). Molecular Phylogenetics and

189 Evolution 57:1276-1292.

190 Drummond AJ, Suchard, MA, Xie D, Rambaut A. 2012. Bayesian phylogenetics with BEAUti

191 and the BEAST 1.7. Molecular Biology and Evolution 29:1969-1973.

192 Drummond AJ, Ho SYW, Phillips MJ, Rambaut A. 2006. Relaxed phylogenetics and dating with 193 confidence. PLoS Biology 4:e88.

194 Edgar RC. 2004. MUSCLE: multiple sequence alignment with high accuracy and high 195 throughput. Nucleic Acids Research 32:1792-1797. 
196 Feng B, Xu J, Wu G, Zeng N-K, Li Y-C, Tolgor B, Kost GW, Yang Z-L. 2012. DNA sequence

197 analyses reveal abundant diversity, endemism and evidence for Asian origin of the porcini

198 mushrooms. PLoS ONE 7:e37567.

199 Fujisawa T, Barraclough TG. 2013. Delimiting species using single-locus data and the

200 generalized mixed Yule coalescent approach: A revised method and evaluation on simulated data

201 sets. Systematic Biology 62:707-724.

202 Galtier N, Gouy M, Gautier C. 1996. SEAVIEW and PHYLO_WIN: two graphic tools for

203 sequence alignment and molecular phylogeny. Computer Applications in the Biosciences 12:543204548.

205 Gardes M, Bruns TD. 1993. ITS primers with enhanced specificity for basidiomycetes -

206 application to the identification of mycorrhizae and rusts. Molecular Ecology 2:113-118.

207 Gouy M, Guindon S, Gascuel O. 2010. SeaView version 4: a multiplatform graphical user

208 interface for sequence alignment and phylogenetic tree building. Molecular Biology and

209 Evolution. 27:221-224.

210 Hebert PDN, Cywinska A, Ball SL, De Waard JR. 2003. Biological identifications through DNA

211 barcodes. Proceedings of the Royal Society of London. Series B, Biological Sciences 270:313-

212321. 
213 Helbling A, Bonadies N, Brander KA, Pichler WJ. 2002. Boletus edulis: a digestion-resistant

214 allergen may be relevant for food allergy. Clinical \& Experimental Allergy 32:771-775.

215 Hibbett DS, Ohman A, Glotzer D, Nuhn M, Kirk PM, Nilsson RH. 2011. Progress in molecular

216 and morphological taxon discovery in Fungi and options for formal classification of

217 environmental sequences. Fungal Biology Reviews 39:147-182.

218 Lücking R, Dal-Forno M, Sikaroodi M, Gillevet PM, Bungartz F, Moncada B, Yánez-Ayabaca A,

219 Chaves JL, Coca LF, Lawrey JD. 2014. A single macrolichen constitutes hundreds of

220 unrecognized species. Proceedings of the National Academy of Sciences of the United States of

221 America. doi: 10.1073/pnas.1403517111

222 Maddison WP, Maddison DR. 2011. Mesquite: a modular system for evolutionary analysis.

223 Version $2.75 \mathrm{http}: / /$ mesquiteproject.org

224 O’Brien HE, Parrent JL, Jackson JA, Moncalvo J-M, Vilgalys R. 2005. Fungal

225 community analysis by large-scale sequencing of environmental samples.

226 Applied and Environmental Microbiology 71: 5544-5550.

227 Ott M, Zola J, Aluru S, Stamatakis A. 2007. Large-scale maximum likelihood-based phylogenetic 228 analysis on the IBM BlueGene/L. Proceedings of ACM/IEEE Supercomputing conference. Article 229 No. 4. 
230 Pons J, Barraclough TG, Gómez-Zurita J, Cardoso A, Duran DP, Hazell S, Kamoun S, Sumlin

231 WD, Vogler AP. 2006. Sequence-based species delimitation for the DNA taxonomy of

232 undescribed insects. Systematic Biology 55:595-609.

233 Reid NM, Carstens BC. 2012. Phylogenetic estimation error can decrease the accuracy of species

234 delimitation: a Bayesian implementation of the general mixed Yule-coalescent model. BMC

235 Evolutionary Biology 12:196.

236 Riedel A, Sagata K, Suhardjono YR, Tänzler R, Balke M. 2013. Integrative taxonomy on the fast

237 track - towards more sustainability in biodiversity research. Frontiers in Zoology 10:15.

238 Sitta N, Floriani M. 2008. Nationalization and globalization trends in the wild mushroom

239 commerce of Italy with emphasis on porcini (Boletus edulis and allied species). Economic

240 Botany 62:307-322.

241 Stamatakis A. 2006. RAxML-VI-HPC: maximum likelihood-based phylogenetic analyses with

242 thousands of taxa and mixed models. Bioinformatics 22:2688.

243 Swofford, D.L., 2002. PAUP*: Phylogenetic Analysis using Parsimony (* and other

244 methods), Version 4.0. Sinauer Associates, Sunderland, MA.

245 Taylor DL, Hollingsworth TN, McFarland JW, Lennon NJ, Nusbaum C, Ruess RW. 2014. A first

246 comprehensive census of fungi in soil reveals both hyperdiversity and fine-scale niche

247 partitioning. Ecological Monographs 84:3-20. 
248 Torricelli R, Johansson SG, Wütrich B. 1997. Ingestive and inhalative allergy to the mushroom 249 Boletus edulis. Allergy 52:747-751.

250 White TJ, Bruns T, Lee S, Taylor J. 1990. Amplification and direct sequencing of fungal 251 ribosomal RNA genes for phylogenetics. In: Innis MA, et al., ed. PCR Protocols: a Guide to 252 Methods and Applications San Diego: Academic Press.

253 Zang M. 2006. Flora fungorum sinicorum: Boletaceae (I). Beijing: Science 254 Press. 
255 Figure 1. Phylogeny and alignment of three unnamed species discovered in a commercial packet

256 of dried porcini. On the left is an ultrametric tree rooted with Boletus aereus and with branch

257 lengths transformed using the uncorrelated relaxed clock model in BEAST. The relationship of

258 the core species or porcini, Boletus edulis, to the dataset is depicted using a dashed line. Clades

259 with dark red branches represent the three maximum likelihood clusters in the GMYC model

260 with the greatest ML score calculated using the single method in the 'splits' package in R.

261 Terminal labels in blue represent sequences derived from individual pieces of mushroom sampled

262 from a commercial packet of porcini. Pie charts on branches show maximum likelihood

263 bootstraps ('MLBS'; lightest red), GMYC supports [19] ('GMYC'; medium red), and posterior

264 probabilities of the cluster as calculated using bGMYC ('bGMYC'; darkest red). On the right is

265 the alignment exported from Mesquite v2.75 (Maddison \& Maddison, 2011) of 34 variable

266 positions in the ITS region after excluding uninformative sites using PAUP* (Swofford, 2002).

267 Nucleotide characters are depicted using IUPAC codes, gaps depicted by a '-' and

268 ambiguous/missing data depicted by '?'.

269 Table 1. Intra- and inter-specific uncorrected ITS barcode sequence distances of the three

270 unnamed species discovered in a commercial packet of dried porcini. Ranges are minimum-

271 maximum distances expressed as percent. 


\section{Figure 1}

Figure 1. Phylogeny and alignment of three unnamed species discovered in a commercial packet of dried porcini.

Figure 1. Phylogeny and alignment of three unnamed species discovered in a commercial packet of dried porcini. On the left is an ultrametric tree rooted with Boletus aereus and with branch lengths transformed using the uncorrelated relaxed clock model in BEAST. The relationship of the core species or porcini, Boletus edulis, to the dataset is depicted using a dashed line. Clades with dark red branches represent the three maximum likelihood clusters in the GMYC model with the greatest ML score calculated using the single method in the 'splits' package in R. Terminal labels in blue represent sequences derived from individual pieces of mushroom sampled from a commercial packet of porcini. Pie charts on branches show maximum likelihood bootstraps ('MLBS'; lightest red), GMYC supports [19] ('GMYC'; medium red), and posterior probabilities of the cluster as calculated using bGMYC ('bGMYC'; darkest red). On the right is the alignment exported from Mesquite v2.75 (Maddison \& Maddison, 2011) of 34 variable positions in the ITS region after excluding uninformative sites using PAUP* (Swofford, 2002). Nucleotide characters are depicted using IUPAC codes, gaps depicted by a '-' and ambiguous/missing data depicted by '?'. 


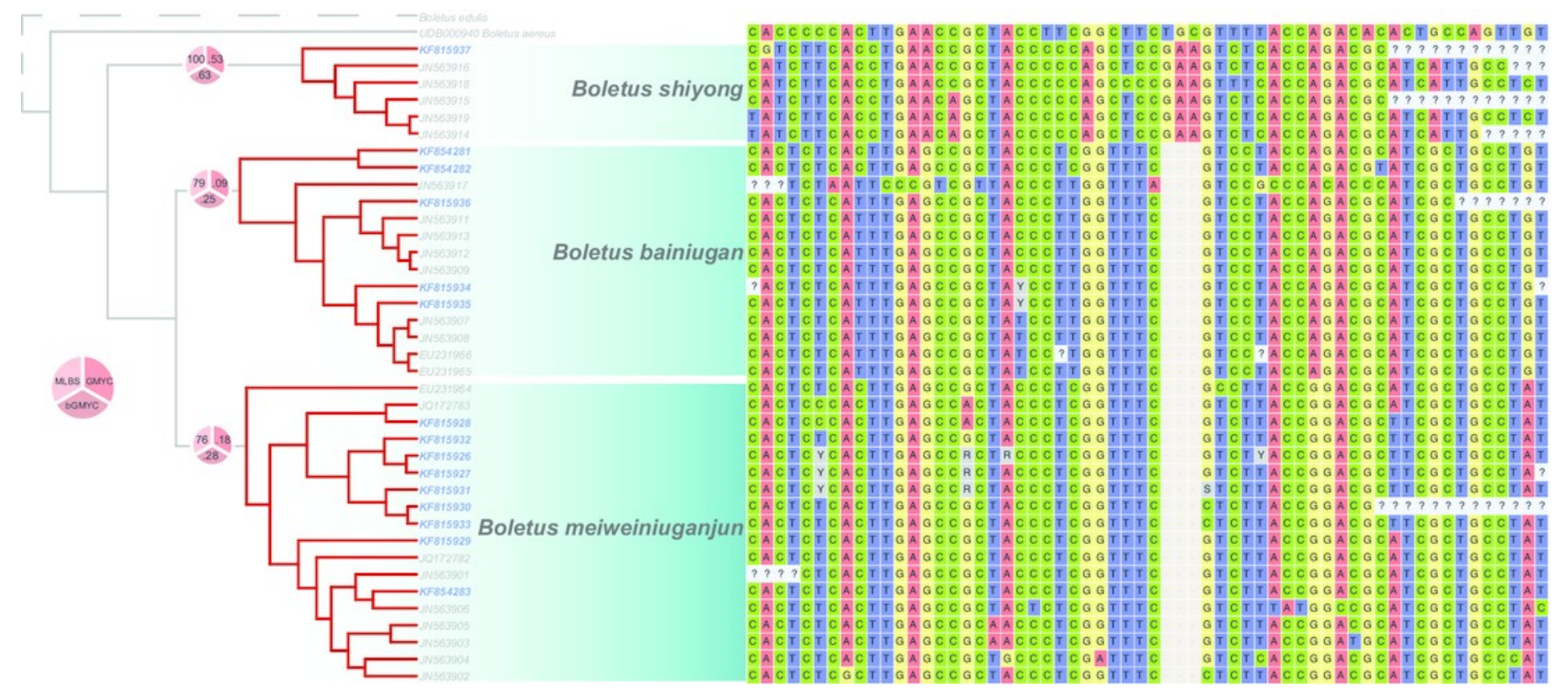




\section{Table $\mathbf{1}_{\text {(on next page) }}$}

Table 1. Intra- and inter-specific uncorrected ITS barcode sequence distances of the three unnamed species discovered in a commercial packet of dried porcini.

Table 1. Intra- and inter-specific uncorrected ITS barcode sequence distances of the three unnamed species discovered in a commercial packet of dried porcini. Ranges are minimummaximum distances expressed as percent. 


\begin{tabular}{lccc}
\hline & B. bainiugan & $\begin{array}{l}\text { B. } \\
\text { meiweiniuganjun }\end{array}$ & B. shiyong \\
$\begin{array}{l}\text { Boletus } \\
\text { bainuigan } \\
\text { B. } \\
\begin{array}{l}\text { meiweiniuganjun } \\
\text { B. shiyong }\end{array}\end{array}$ & $0-2.2$ & $0-1.5$ & \\
\hline
\end{tabular}

